Artículo de reflexión no derivado de investigación

\title{
Inteligencia emocional y práctica clínica en tiempos de pandemia
}

\section{Emotional intelligence and clinical practice in pandemic times}

\author{
Sara Sierra- Tobón ${ }^{1 \otimes C \text { CvLAC }}$, Carlos E. Díaz-Castrillón ${ }^{2}$ CvLAC
}

Fecha correspondencia:

Recibido: mayo 11 de 2020. Revisado: julio 31 de 2020. Aceptado: agosto 4 de 2020.

Forma de citar:

Sierra-Tobón S, Díaz-Castrillón CE. Inteligencia emocional y práctica clínica en tiempos de pandemia. Rev CES Med. 2020; Especial COVID-19: 59-68.

Open access

(C) Derecho de autor

Licencia creative commons

Ética de publicaciones

Revisión por pares

Gestión por Open Journal System

DOl: http://dx.doi.org/10.21615/

cesmedicina.34.COVID-19.9

ISSN 0120-8705

e-ISSN 2215-9177

Sobre los autores:

1. Especialista en Anestesiología, Coach en Salud y Nutrición Integrativa, Postdoctoral Research Fellow, University of Illinois at Chicago.

Comparte

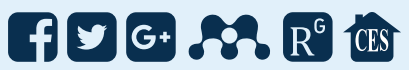

\section{Resumen}

Los cambios generados por la pandemia COVID-19 han significado innumerables retos en la práctica clínica, en los que el personal de salud ha tenido que adaptarse profesional y emocionalmente. Fortalecer la inteligencia emocional en los profesionales de la salud contribuye a su bienestar personal, previene el síndrome de burnout y ayuda a la creación de ambientes laborales seguros, objetivos fundamentales a la hora de enfrentar desafíos como la pandemia actual. Cuatro habilidades de la inteligencia emocional que son particularmente importantes en la práctica clínica (autoconciencia, autorregulación, conciencia social y manejo de relaciones) son objeto de reflexión en el presente artículo.

Palabras clave: Inteligencia emocional; Burnout; Profesional de la salud; Pandemia; COVID-19.

\begin{abstract}
The changes generated by the COVID-19 pandemic have meant countless challenges in clinical practice, in which health personnel have had to adapt professionally and emotionally. Four skills in emotional intelligence that are particularly important in clinical practice (self-awareness, self-regulation, social awareness and relationship management) are the subject of reflection in this article. Strengthening emotional intelligence in health professionals contributes to their personal well-being, prevents burnout syndrome and helps create safe working environments, which are fundamental objectives when facing challenges such as the current pandemic.
\end{abstract}

Keywords: Emotional intelligence; Burnout; Health professional; Pandemic; COVID-19.

\section{Introducción}

La práctica clínica es un escenario complejo y emocionalmente desafiante, especialmente cuando la pandemia por SARS-CoV-2 ha llevado a los trabajadores de la salud a enfrentar circunstancias sin precedentes en la historia de la Medicina moderna. Como consecuencia, la capacidad de resiliencia de los diferentes actores es puesta a prueba constantemente, con potenciales implicazciones psicológicas y clínicas a largo plazo (1-3).

Existen razones para reconocer al personal de la salud como población vulnerable en situaciones como esta. En primer lugar hay una mayor proporción de personal asistencial afectado por el síndrome de burnout en 
2. Especialista en Cirugía General, Posdoctoral Research Fellow, University of Pittsburgh Medical Center.

Existen cuatro atributos de la inteligencia emocional que resultan de especial relevancia en la práctica clínica y agrupados en dos categorías: atributos individuales y atributos sociales. En los primeros se incluyen la autoconciencia y la autorregulación. Dentro de los atributos sociales se encuentran la conciencia social y el manejo de las relaciones. comparación con la población general, estando presente en 30-70\% de médicos y enfermeras (4). En segundo lugar, porque se ha observado un aumento relativo en la incidencia de síndrome de burnout y otros trastornos mentales en el personal de salud durante brotes epidémicos previos (5).

En consecuencia, temas como la inteligencia emocional y su relación con el desempeño profesional y la seguridad del paciente, tienen cada vez mayor relevancia en el mundo académico y en la formación de futuros profesionales de la salud. A continuación, describimos la relación de cuatro habilidades de la inteligencia emocional con el bienestar del personal de la salud y el desempeño profesional.

\section{Definiendo la inteligencia emocional, el síndrome de burnout y cómo se relacionan con la seguridad del paciente}

Si bien desde los años 60 se habla de inteligencia emocional, fue en la década de 1990 cuando los sicólogos norteamericanos Peter Salovey y Jhon D. Mayer propusieron la idea de un conjunto de habilidades que son necesarias para la adecuada identificación y expresión emocional de los seres humanos (6). La inteligencia emocional se define como la capacidad de reconocer, entender y controlar las emociones propias y el impacto que pueden tener en los demás, usando la información que proveen para orientar nuestros pensamientos y acciones $(6,7)$. Otros autores, como Reuven Bar-On y Daniel Goleman han expandido el concepto, considerando la inteligencia emocional como un conjunto de habilidades que pueden ser aprendidas y fortalecidas $(8,9)$.

Existen cuatro atributos de la inteligencia emocional que resultan de especial relevancia en la práctica clínica (10), los cuales están agrupados en dos categorías: atributos individuales y atributos sociales (9). En los primeros se incluyen la autoconciencia -entendida como la capacidad de reconocer los estados de ánimo propios, fortalezas, debilidades, motivaciones y metas. La autorregulación -la capacidad de mantener la calma ante emociones fuertes y usarlas de forma constructiva y no impulsiva-. Dentro de los atributos sociales se encuentran la conciencia social -capacidad de identificar y entender las emociones, necesidades y preocupaciones de los demás,- y, el manejo de relaciones, que consiste en la capacidad de construir vínculos saludables por medio de una comunicación asertiva que permita tener influencia en otros (11). Adicionalmente, se han desarrollado herramientas de medición para determinar qué tan desarrolladas están dichas habilidades en las personas, lo cual recibe el nombre de coeficiente emocional (EQ) (12). Si bien pueden tener algunas diferencias, dichos instrumentos de medición concuerdan en determinar que tan hábil es una persona para percibir, regular y utilizar las emociones (13).

El burnout es un síndrome sicológico que emerge como respuesta al estrés laboral crónico y comprende tres dimensiones claves: sensación de agotamiento, sentimientos negativos o cínicos con respecto al trabajo y menor desempeño profesional (14). En este modelo tridimensional la experiencia estresante del individuo se desarrolla en un contexto social con los demás pares, en el que influye la concepción que se tiene de sí mismo y de los demás (15). La relevancia clínica de este fenómeno reside en la asociación con desenlaces desfavorables como mayor riesgo de cometer errores en la toma de decisiones clínicas (16), menor productividad laboral (17) e insatisfacción de los pacientes (18). La prevalencia de burnout en los profesionales de la salud se estima alrededor del 50\% (19), alcanzando niveles más altos en personal médico en formación (20). 
Las emociones son un determinante potente y común en los procesos de juicio y en situaciones de conflicto este proceso cognitivo se ve afectado. Sin embargo, incluso las decisiones clínicas en el día a día se toman en contextos emocionalmente desafiantes, lo cual requiere profesionales que sean conscientes de sus emociones y las de los demás.
Se ha observado que aquellos trabajadores de la salud que están motivados a regular sus emociones y tienen un sentido de empatía desarrollado, tienen menor riesgo de experimentar insatisfacción laboral, lo que a su vez, se refleja en una mejor atención a los pacientes (21). Sahid et al. demuestran que las intervenciones educativas que buscan mejorar habilidades de autoconciencia, autorregulación, conciencia social y manejo de relaciones en médicos residentes se asocian con mayor percepción de bienestar y menor incidencia de síndrome de burnout (22); al igual que existe una relación inversamente proporcional entre el coeficiente emocional y la incidencia de agotamiento emocional entre residentes de cirugía general (23).

El mejor desempeño profesional de aquellos con alto coeficiente emocional puede deberse en parte a que el proceso de toma de decisiones clínicas está influenciado por el tono emocional del médico (24). Las emociones son un determinante potente y común en los procesos de juicio y en situaciones de conflicto este proceso cognitivo se ve afectado $(25,26)$. Sin embargo, incluso las decisiones clínicas en el día a día se toman en contextos emocionalmente desafiantes, lo cual requiere profesionales que sean conscientes de sus emociones y las de los demás. Por ejemplo, el dar una mala noticia al paciente es un evento percibido como estresante y se asocia con activación simpática y dificultad en el manejo de emociones como perdida, culpa y fracaso (27).

En una revisión publicada en 2016, Heyhoe et al. plantean que las emociones de los prestadores impactan la seguridad del paciente por tres razones: la presencia /ausencia de empatía hacia el paciente puede influir en la decisión terapéutica; un ambiente laboral hostil y conflictos con los colegas puede generar sentimientos que afectan el juicio individual y colectivo; y, las experiencias previas similares determinan una respuesta emocional y con ello el juicio sobre qué tanta información se requiere para llegar a una decisión clínica (28). Es decir, que la inteligencia emocional puede considerarse un factor a favor de la seguridad del paciente en la medida que su fortalecimiento contribuye al bienestar del profesional de la salud y a su vez, al desarrollo de un ambiente seguro para los pacientes.

\section{Habilidades de autoconciencia y autorregulación para sentirnos menos fuera de control}

Durante los últimos meses nos hemos enfrentado a emociones que son difíciles de ignorar. El cambio súbito en nuestro entorno laboral, tanto de las instalaciones físicas como la forma de relacionamos generan sentimientos de extrañeza e incertidumbre; los trabajadores de la salud tenemos la preocupación de adquirir la enfermedad y contagiar a nuestros seres queridos; así, por ejemplo, 65\% de los cirujanos encuestados durante el inicio de la fase de confinamiento de la pandemia en Colombia consideraron que las recomendaciones de bioseguridad para procedimientos en pacientes sospechosos de infección se cumplían solo de manera parcial (29). También está el temor a ser estigmatizado y muchas veces maltratado física y sicológicamente por parte de la comunidad. Todo lo anterior hace que el día laboral sea emocionalmente intenso; sin embargo, el reconocimiento de lo que se está sintiendo, la reacción ante dichos sentimientos y evaluar cómo esto puede afectar su desempeño, es el primer paso para tener mayor control.

Las investigaciones coinciden en que cuando apartamos, negamos o ignoramos lo que sentimos, no resolvemos nuestros problemas (30). El primer paso para conectarnos con nuestra inteligencia emocional es reconocer nuestros sentimientos, sin juicio y sin tomar acción. Imágenes de resonancia magnética funcional han demostrado cómo al 
Es importante recordar que la capacidad de tomar la mejor decisión en un momento difícil radica en ese espacio que yace entre el estímulo y la respuesta. darle nombre a las emociones, la actividad neuronal aumenta en la corteza frontal donde se llevan a cabo procesos racionales y se atenúa la actividad límbica, lo cual genera una sensación de calma y auto control ante emociones difíciles y disminuye su intensidad (31)

En la práctica clínica, el énfasis en el paciente hace que muchas veces el profesional de salud olvide prestar atención a sus necesidades básicas y a sus emociones. Para ello incluir pausas cortas durante el día como micro-prácticas de bienestar puede ser de ayuda (32). Aproveche estas pausas para preguntarse: ¿Qué estoy sintiendo?, ¿tengo mis necesidades básicas satisfechas?, ¿en qué tono me estoy hablando a mi mismo?, ¿soy consciente de cómo estoy hablando a los demás y como esto puede afectarlos?

Estas prácticas de autoconciencia son especialmente importantes en espacios hospitalarios donde se trabaja en equipo, dado que el líder tiene gran influencia en el tono emocional de los integrantes y está demostrado que la inteligencia emocional del líder se relaciona directamente con la efectividad del grupo (33).

Una vez se logra identificar y reivindicar los sentimientos y necesidades propias, se cuenta con la información necesaria para regularlos y controlar las respuestas ante los estímulos del medio. Ese proceso de autorregulación voluntaria de las emociones tiene lugar en la corteza frontal, una región cerebral crítica para la generación, expresión y experiencia de las emociones negativas (34) e influye en la actividad de la amígdala evitando que en ante un evento detonante se pierda el control.

En este contexto es importante recordar que la capacidad de tomar la mejor decisión en un momento difícil radica en ese espacio que yace entre el estimulo y la respuesta (35). Al identificar un evento detonante deténgase un momento y permita que su sistema nervioso y sus respuestas fisiológicas se apacigüen; respirar profundo varias veces; entrar en movimiento y la música son herramientas que ayudan a regular las emociones y atenúan la descarga simpática (36-38). Utilice micro-pausas de bienestar para focalizar su atención en aquellas cosas que están en su zona de influencia, las decisiones que debe tomar hoy y el paciente que está frente a usted. Esto le ayudará a convertir la sensación de pérdida de control en una serie de acciones ejecutables, que una vez realizadas se traducirán en sensación de alivio y satisfacción (39).

Ser autocompasivos también es importante, separar espacio para el descanso, la alimentación y el ejercicio contribuyen a fortalecer la resiliencia frente a los retos laborales y personales (40), como también lo hace el permitirse recibir apoyo de su familia, colegas y profesionales de la salud mental para lidiar con el día a día en las circunstancias actuales (41). Permítase un momento para preguntarse: ¿Puedo reconocer aquellas cosas que me detonan?, ¿cuáles son algunas cosas en mi vida que puedo controlar?, ¿estoy cuidando de mi permitiéndome descansar, alimentarme y ejercitarme?, ¿necesito apoyo para asumir los retos que enfrento hoy? 
Mayor empatía por parte del médico hacia el paciente se relaciona con mayor adherencia terapéutica y mejores desenlaces, mientras también contribuye a la prevención y resolución de conflictos en la práctica clínica.

\section{Habilidades de conciencia social y manejo de relaciones de cara a la adversidad}

Una vez el profesional de la salud cuida de si mismo mediante la autoconciencia y la autorregulación, estará en capacidad de cuidar a otros. El tener empatía por los colegas, pacientes y familiares es fundamental especialmente en circunstancias como las que se viven actualmente. La empatía caracteriza la conciencia social y es el atributo de la inteligencia emocional que permite reconocer y validar las emociones y necesidades de los demás (42).

Mayor empatía por parte del médico hacia el paciente se relaciona con mayor adherencia terapéutica y mejores desenlaces (43), mientras también contribuye a la prevención y resolución de conflictos en la práctica clínica (44). Tener en cuenta las emociones de los demás y cómo sus respuestas y acciones influyen en ellas le permitirá identificar la mejor manera de proceder, de forma que sus relaciones laborales sean productivas. Podemos incrementar la empatía mediante la escucha atenta del otro identificando sus necesidades en un momento determinado (45), lo cual permitirá tener una comunicación clínica efectiva, caracterizada por ser respetuosa, clara, directa y explicita que contribuya a la seguridad del paciente (46).

Para fortalecer su conciencia social y empatía pregúntese: ¿Realmente estoy escuchando a los demás para entenderlos o solo para saber que responder?, ¿Puedo identificar y nombrar las emociones de los demás?, ¿hay alguien cuyo trabajo no he reconocido hoy?

Una vez se tiene conciencia social se puede usar dicha información para tener interacciones sociales asertivas que le permitan motivar e influenciar a otros, lo cual se traduce en un mejor manejo de relaciones (47). Dado el aumento en la carga laboral y la ansiedad por la pandemia, el manejo de relaciones puede ser particularmente desafiante en el ámbito hospitalario; además, es común encontrarse con personal que ha sido reasignado a otras áreas y con el cual nunca se ha trabajado antes, siendo preciso fortalecer dichas relaciones para garantizar la productividad del equipo.

En cuanto a los pacientes, en el contexto de COVID-19 el manejo de relaciones también resulta fundamental, ya que la mayoría de pacientes se cuidarán a sí mismos en casa y un paciente satisfecho tendrá mayor adherencia al tratamiento y las medidas de protección las cuales son cruciales para el control de la enfermedad $(48,49)$.

Ser paciente en las interacciones con el equipo y los pacientes, prestar atención al lenguaje corporal y expresiones faciales de forma que inspiren confianza y atención, son formas de fortalecer las relaciones con los demás.

Como líder que es, trate de incluir en estos momentos expresiones como "Hagamos esto despacio porque es importante" y "Gracias". Para tener mejores relaciones con los demás en momentos difíciles puede preguntarse: ¿Estoy transmitiendo paciencia y asumiendo que los demás están haciendo su mejor esfuerzo?, ¿como líder, estoy siendo transparente con la información?, ¿es la comunicación con mi equipo frecuente, clara y abierta a la retroalimentación? 
Desde la inteligencia emocional podemos desarrollar mayor tolerancia al estrés, preservar el bienestar individual y crear ambientes laborales seguros para el paciente. Aplicamos la inteligencia emocional cuando afrontamos los desafíos de la práctica clínica desde el auto cuidado, con empatía, apertura y disposición a trabajar en equipo.

\section{Conclusiones}

Si bien la pandemia por COVID- 19 ha sido una gran prueba para los profesionales de la salud, también presenta una oportunidad para fortalecernos como individuos y como grupo. La resiliencia que se requiere para enfrentar circunstancias como las actuales es un proceso dinámico y en constante evolución que requiere no solo de una actitud positiva sino también de estrategias efectivas. El bienestar de los profesionales de la salud es un elemento fundamental a la hora de desarrollar estrategias para afrontar la pandemia que garanticen la integridad del acto médico y los actores involucrados. Desde la inteligencia emocional podemos desarrollar mayor tolerancia al estrés, preservar el bienestar individual y crear ambientes laborales seguros para el paciente. Aplicamos la inteligencia emocional cuando afrontamos los desafíos de la práctica clínica desde el auto cuidado, con empatía, apertura y disposición a trabajar en equipo.

\section{Conflicto de intereses}

Los autores declaran no tener ningún conflicto de intereses en la publicación de este artículo.

\section{Bibliografía}

1. Liu Q, Luo D, Haase JE, Guo Q, Wang XQ, Liu S, et al. The experiences of health-care providers during the COVID-19 crisis in China: a qualitative study. Lancet Glob Heal [Internet]. 2020 Apr; Available from: https://linkinghub.elsevier.com/retrie$\mathrm{ve/pii/S2214109 \times 20302047}$

2. Lai J, Ma S, Wang Y, Cai Z, Hu J, Wei N, et al. Factors Associated With Mental Health Outcomes Among Health Care Workers Exposed to Coronavirus Disease 2019. JAMA Netw Open [Internet]. 2020 Mar 23;3 (3):e203976. Available from: https:// jamanetwork.com/journals/jamanetworkopen/fullarticle/2763229

3. Kang L, Li Y, Hu S, Chen M, Yang C, Yang BX, et al. The mental health of medical workers in Wuhan, China dealing with the 2019 novel coronavirus. The Lancet Psychiatry [Internet]. 2020 Mar 1 [cited 2020 May 5];7 (3):e14. Available from: http://www.ncbi.nlm.nih.gov/pubmed/32035030

4. Hall LH, Johnson J, Watt I, Tsipa A, O'Connor DB. Healthcare Staff Wellbeing, Burnout, and Patient Safety: A Systematic Review. Harris F, editor. PLoS One [Internet]. 2016 Jul 8;11 (7):e0159015. Available from: https://dx.plos.org/10.1371/ journal.pone.0159015

5. Maunder R, Lancee W, Balderson K, Bennett J, Borgundvaag B, Evans S, et al. Long-term Psychological and Occupational Effects of Providing Hospital Healthcare during SARS Outbreak. Emerg Infect Dis [Internet]. 2006;12 (12):1924-32. Available from: http://wwwnc.cdc.gov/eid/article/12/12/06-0584 article.htm

6. Salovey P, Mayer JD. Emotional Intelligence. Imagin Cogn Pers [Internet]. 1990 Mar 28;9 (3):185-211. Available from: http://journals.sagepub.com/doi/10.2190/ DUGG-P24E-52WK-6CDG

7. Mayer JD, Salovey P, Caruso DR. Emotional intelligence: New ability or eclectic traits? Am Psychol [Internet]. 2008;63 (6):503-17. Available from: http://doi.apa. org/getdoi.cfm?doi=10.1037/0003-066X.63.6.503 
8. Bar-On R. The Bar-On model of emotional-social intelligence (ESI). Psicothema [Internet]. 2006;18 Suppl:13-25. Available from: http://www.ncbi.nlm.nih.gov/ pubmed/17295953

9. Goleman D. What makes a leader? Harv Bus Rev [Internet]. 76 (6):93-102. Available from: http://www.ncbi.nlm.nih.gov/pubmed/10187249

10. Mintz LJ, Stoller JK. A Systematic Review of Physician Leadership and Emotional Intelligence. J Grad Med Educ [Internet]. 2014 Mar;6 (1):21-31. Available from: http://www.jgme.org/doi/abs/10.4300/JGME-D-13-00012.1

11. Mayer JD, Salovey P. The intelligence of emotional intelligence. Intelligence [Internet]. 1993 Oct;17 (4):433-42. Available from: https://linkinghub.elsevier.com/ retrieve/pii/0160289693900103

12. Mayer JD, Salovey P, Caruso DR, Sitarenios G. Measuring emotional intelligence with the MSCEIT V2.0. Emotion [Internet]. 2003:3 (1):97-105. Available from: http://doi.apa.org/getdoi.cfm?doi=10.1037/1528-3542.3.1.97

13. O'Connor PJ, Hill A, Kaya M, Martin B. The Measurement of Emotional Intelligence: A Critical Review of the Literature and Recommendations for Researchers and Practitioners. Front Psychol [Internet]. 2019 May 28;10. Available from: https:// www.frontiersin.org/article/10.3389/fpsyg.2019.01116/full

14. World Health Organization. International classification of diseases for mortality and morbidity statistics (11th Revision). [Internet]. 2018. Available from: https:// icd.who.int/browse11/l-m/

15. Maslach C, Leiter MP. Understanding the burnout experience: recent research and its implications for psychiatry. World Psychiatry [Internet]. 2016 Jun;15 (2):103-11. Available from: http://doi.wiley.com/10.1002/wps.20311

16. Birks YF, Watt IS. Emotional intelligence and patient-centred care. J R Soc Med [Internet]. 2007 Aug;100 (8):368-74. Available from: http://www.ncbi.nlm.nih. gov/pubmed/17682030

17. Dewa CS, Loong D, Bonato S, Thanh NX, Jacobs P. How does burnout affect physician productivity? A systematic literature review. BMC Health Serv Res [Internet] 2014 Dec 28:14 (1):325. Available from: https://bmchealthservres.biomedcentral.com/articles/10.1186/1472-6963-14-325

18. Weng H-C. Does the physician's emotional intelligence matter? Impacts of the physician's emotional intelligence on the trust, patient-physician relationship, and satisfaction. Health Care Manage Rev [Internet]. 33 (4):280-8. Available from: http://www.ncbi.nlm.nih.gov/pubmed/18815493

19. Rotenstein LS, Torre M, Ramos MA, Rosales RC, Guille C, Sen S, et al. Prevalence of Burnout Among Physicians. JAMA [Internet]. 2018 Sep 18;320 (11):1131. Available from: http://jama.jamanetwork.com/article.aspx?doi=10.1001/ jama.2018.12777 
20. IsHak WW, Lederer S, Mandili C, Nikravesh R, Seligman L, Vasa M, et al. Burnout During Residency Training: A Literature Review. J Grad Med Educ [Internet]. 2009 Dec;1 (2):236-42. Available from: http://www.jgme.org/doi/abs/10.4300/ JGME-D-09-00054.1

21. Bulmer Smith K, Profetto-McGrath J, Cummings GG. Emotional intelligence and nursing: an integrative literature review. Int J Nurs Stud [Internet]. 2009 Dec;46 (12):1624-36. Available from: http://www.ncbi.nlm.nih.gov/pubmed/19596323

22. Shahid R, Stirling J, Adams W. Promoting wellness and stress management in residents through emotional intelligence training. Adv Med Educ Pract [Internet]. 2018 Sep;Volume 9:681-6. Available from: https://www.dovepress.com/ promoting-wellness-and-stress-management-in-residents-through-emotiona-peer-reviewed-article-AMEP

23. Lin DT, Liebert CA, Tran J, Lau JN, Salles A. Emotional Intelligence as a Predictor of Resident Well-Being. J Am Coll Surg [Internet]. 2016 Aug;223 (2):352-8. Available from: https://linkinghub.elsevier.com/retrieve/pii/S1072751516301429

24. Kozlowski D, Hutchinson M, Hurley J, Rowley J, Sutherland J. The role of emotion in clinical decision making: an integrative literature review. BMC Med Educ [Internet]. 2017 Dec 15;17 (1):255. Available from: https://bmcmededuc.biomedcentral.com/articles/10.1186/s12909-017-1089-7

25. Lerner JS, Li Y, Valdesolo P, Kassam KS. Emotion and Decision Making. Annu Rev Psychol [Internet]. 2015 Jan 3;66 (1):799-823. Available from: http://www. annualreviews.org/doi/10.1146/annurev-psych-010213-115043

26. Garfinkel SN, Zorab E, Navaratnam N, Engels M, Mallorquí-Bagué N, Minati L, et al. Anger in brain and body: the neural and physiological perturbation of decision-making by emotion. Soc Cogn Affect Neurosci [Internet]. 2016 Jan 1;11 (1):150-8. Available from: https://academic.oup.com/scan/article/11/1/150/2375178

27. Fallowfield $L$, Jenkins V. Communicating sad, bad, and difficult news in medicine. Lancet [Internet]. 2004 Jan;363 (9405):312-9. Available from: https://linkinghub. elsevier.com/retrieve/pii/S0140673603153925

28. Heyhoe J, Birks Y, Harrison R, O'Hara JK, Cracknell A, Lawton R. The role of emotion in patient safety: Are we brave enough to scratch beneath the surface? J R Soc Med [Internet]. 2016 Feb 18;109 (2):52-8. Available from: http://journals. sagepub.com/doi/10.1177/0141076815620614

29. Díaz-Castrillón CE, Cortés N, Rey S, Pineda M, Díaz-Castrillón JF, Sierra Tobón S. Percepción de la Pandemia COVID-19 en los Servicios de Cirugía en Colombia. Rev Colomb Cirugía [Internet]. 2020 May 11;35 (2):290-301. Available from: https://www.revistacirugia.org/index.php/cirugia/article/view/655

30. Barrett LF, Gross J, Christensen TC, Benvenuto M. Knowing what you're feeling and knowing what to do about it: Mapping the relation between emotion differentiation and emotion regulation. Cogn Emot [Internet]. 2001 Nov;15 (6):713-24. Available from: http://www.tandfonline.com/doi/abs/10.1080/02699930143000239 
31. Lieberman MD, Eisenberger NI, Crockett MJ, Tom SM, Pfeifer JH, Way BM. Putting feelings into words: affect labeling disrupts amygdala activity in response to affective stimuli. Psychol Sci [Internet]. 2007 May;18 (5):421-8. Available from: http://www.ncbi.nlm.nih.gov/pubmed/17576282

32. Fessell D, Cherniss C. Coronavirus Disease 2019 (COVID-19) and Beyond: Micropractices for Burnout Prevention and Emotional Wellness. J Am Coll Radiol [Internet]. 2020 Mar; Available from: https://linkinghub.elsevier.com/retrieve/pii/ $\underline{\mathrm{S} 1546144020302908}$

33. Melita Prati L, Douglas C, Ferris GR, Ammeter AP, Buckley MR. EMOTIONAL INTELLIGENCE, LEADERSHIP EFFECTIVENESS, AND TEAM OUTCOMES. Int J Organ Anal [Internet]. 2003 Jan;11 (1):21-40. Available from: https://www.emerald. com/insight/content/doi/10.1108/eb028961/full/html

34. Beauregard M, Lévesque J, Bourgouin P. Neural Correlates of Conscious Self-Regulation of Emotion. J Neurosci [Internet]. 2001 Sep 15;21 (18):RC165-RC165. Available from: http://www.jneurosci.org/lookup/doi/10.1523/JNEUROSCl.21-18-j0001.2001

35. Gupta R, Koscik TR, Bechara A, Tranel D. The amygdala and decision-making. Neuropsychologia [Internet]. 2011 Mar;49 (4):760-6. Available from: https://linkinghub.elsevier.com/retrieve/pii/S0028393210004197

36. Arch JJ, Craske MG. Mechanisms of mindfulness: Emotion regulation following a focused breathing induction. Behav Res Ther [Internet]. 2006 Dec;44 (12):1849-58. Available from: https://linkinghub.elsevier.com/retrieve/pii/ $\underline{\text { S0005796705002743 }}$

37. Shafir T, Tsachor RP, Welch KB. Emotion Regulation through Movement: Unique Sets of Movement Characteristics are Associated with and Enhance Basic Emotions. Front Psychol [Internet]. 2016 Jan 11;6. Available from: http://journal.frontiersin.org/Article/10.3389/fpsyg.2015.02030/abstract

38. Saarikallio S. Music as emotional self-regulation throughout adulthood. Psychol Music [Internet]. 2011 Jul;39 (3):307-27. Available from: http://journals.sagepub. $\mathrm{com} / \mathrm{doi} / 10.1177 / 0305735610374894$

39. Wood W, Rünger D. Psychology of Habit. Annu Rev Psychol [Internet]. 2016 Jan 4;67 (1):289-314. Available from: http://www.annualreviews.org/doi/10.1146/ annurev-psych-122414-033417

40. Brennan EJ. Towards resilience and wellbeing in nurses. $\mathrm{Br} J$ Nurs [Internet]. 2017 Jan 12;26 (1):43-7. Available from: http://www.magonlinelibrary.com/ doi/10.12968/bjon.2017.26.1.43

41. Jun J, Tucker S, Melnyk BM. Clinician Mental Health and Well-Being During Global Healthcare Crises: Evidence Learned From Prior Epidemics for COVID-19 Pandemic. Worldviews Evidence-Based Nurs [Internet]. 2020 Jun 22;17 (3):182-4. Available from: https://onlinelibrary.wiley.com/doi/abs/10.1111/wvn.12439 
42. Skinner C, Spurgeon P. Valuing empathy and emotional intelligence in health leadership: a study of empathy, leadership behaviour and outcome effectiveness. Heal Serv Manag Res [Internet]. 2005 Feb 21;18 (1):1-12. Available from: http:// journals.sagepub.com/doi/10.1258/0951484053051924

43. Hojat M, Louis DZ, Markham FW, Wender R, Rabinowitz C, Gonnella JS. Physicians' Empathy and Clinical Outcomes for Diabetic Patients. Acad Med [Internet]. 2011 Mar;86 (3):359-64. Available from: http://journals.lww.com/00001888$\underline{201103000-00026}$

44. MORRISON J. The relationship between emotional intelligence competencies and preferred conflict-handling styles. J Nurs Manag [Internet]. 2008 Nov;16 (8):97483. Available from: http://doi.wiley.com/10.1111/j.1365-2834.2008.00876.x

45. Halpern J. What is clinical empathy? J Gen Intern Med [Internet]. 2003 Aug;18 (8):670-4. Available from: http://link.springer.com/10.1046/j.15251497.2003.21017.x

46. Lyndon A, Zlatnik MG, Wachter RM. Effective physician-nurse communication: a patient safety essential for labor and delivery. Am J Obstet Gynecol [Internet]. 2011 Aug;205 (2):91-6. Available from: https://linkinghub.elsevier.com/retrieve/ pii/S0002937811004686

47. Prezerakos PE. Nurse Managers' Emotional Intelligence and Effective Leadership: A Review of the Current Evidence. Open Nurs J [Internet]. 2018 May 31;12 (1):86-92. Available from: https://opennursingjournal.com/VOLUME/12/ PAGE/86/

48. Dias-Barbosa C, Balp, Kulich K, Germain N, Rofail D. A literature review to explore the link between treatment satisfaction and adherence, compliance, and persistence. Patient Prefer Adherence [Internet]. 2012 Jan;39. Available from: http:// www.dovepress.com/a-literature-review-to-explore-the-link-between-treatment-satisfaction-peer-reviewed-article-PPA

49. Lotfi M, Hamblin MR, Rezaei N. COVID-19: Transmission, prevention, and potential therapeutic opportunities. Clin Chim Acta [Internet]. 2020 Sep;508:254-66. Available from: https://linkinghub.elsevier.com/retrieve/pii/S0009898120302503 